\title{
THE DISTRIBUTION OF NEUTRAL HYDROGEN IN THE ORION REGION
}

\author{
H. VAN WOERDEN \\ Kapteyn Astronomical Laboratory, Groningen, The Netherlands
}

Neutral hydrogen is the main constituent of interstellar material. While solid particles and ionized hydrogen have long been studied, detailed investigations of the distribution of neutral hydrogen have only recently become possible. The first survey of neutral hydrogen in Orion was made by Menon $[1,2]$ with the Harvard 24-foot telescope and reported at Jodrell Bank in 1955. A more detailed study of the structure and dynamics of the interstellar medium in this region is now in progress with the 25-meter reflector at Dwingeloo. Its beamwidth is 0.57 at $21 \mathrm{~cm}$, corresponding to 5 parsecs at the distance of the Orion association. The bandwidth of the receiver was $20 \mathrm{kc} / \mathrm{s}$, or $4 \mathrm{~km} / \mathrm{second}$, in this program. Observations with $10 \mathrm{kc} / \mathrm{s}$ bandwidth are being added.

Our study has been centered on the region between right ascensions $5^{\mathrm{h}} 10^{\mathrm{m}}$ and $5^{\mathrm{b}} 50^{\mathrm{m}}$, and declinations 0 and -7 degrees. A few profiles have been taken outside this area, especially along the lines $b=-17.5, l=172.5$, and $l=175: 0$. We plan to extend the study of the surroundings, with a view to disentangling the Orion radiation from its fore- and background.

Over 400 profiles at about 250 different positions have been obtained so far. Since only part of them have been reduced, we limit ourselves to a preliminary discussion of some interesting features.

In broad lines, Menon's earlier work is nicely confirmed by our profiles, which apparently support his conclusion about the gas expansion in the Barnard ring. Of course, finer details in the spatial and velocity distributions have since been found, but which refinements in Menon's dynamical picture are needed is still uncertain.

Examples of our results are shown in Figs. 1, 2, and 3. In the complicated region between the belt and the sword (Fig. 1), the profiles change rapidly over distances of the order of $0: 8=7$ parsecs. This region includes the $\sigma$ Orionis $\mathrm{H} I \mathrm{I}$ region and the Horsehead nebula. The region north of $\beta$ Orionis (Fig. 2) is optically less interesting, but the hydrogen profiles show the existence, even at these latitudes, of several clouds about two degrees in size.

Profiles taken in three fields southwest of the Orion nebula are shown in Fig. 3. The hydrogen profiles at the two lower positions are about equal; the top profile, at only $0: 6=5$ parsecs distance from these two, contains much stronger radiation. A Lick photograph (Fig. 4) shows that the latter position is located in a heavy-absorption region, in which the Orion nebula is embedded; the other two fields are relatively free from absorption as indicated by the numbers of stars and extragalactic nebulae. 


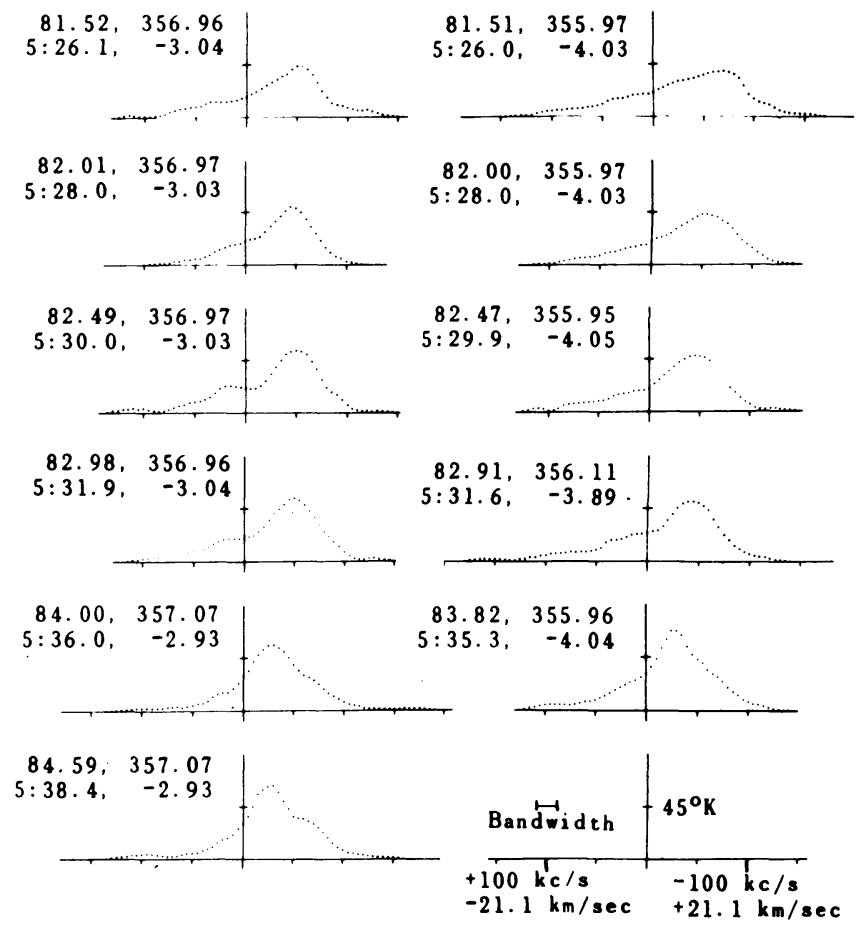

FIG. 1. Profiles obtained in the Orion region, between the belt and the sword. The profiles are arranged in order of right ascension (increasing downward) and declination (increasing to the left). Right ascension and declination are shown in the upper left corner of each profile figure, in telescope coordinates (degrees) and on the conventional system. Scales of frequency, radial velocity, and intensity are shown in the bottom right figure.

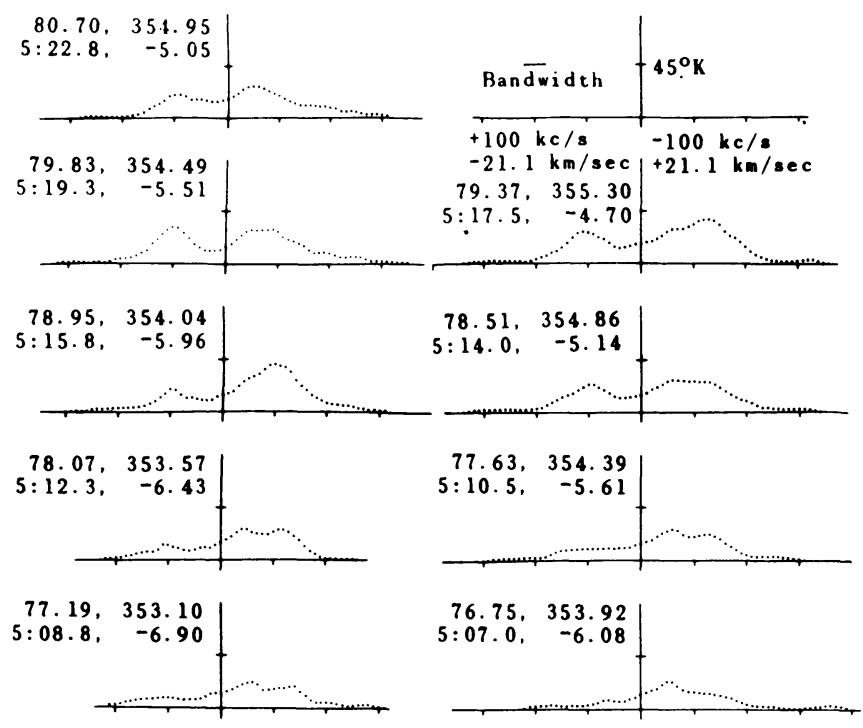

FIG. 2. Profiles obtained in the south preceding part of the Orion region, about 3 degrees north of $\beta$ Orionis. The profiles are arranged along lines of constant $b$ ( -20 degrees at top, -24 degrees at bottom) and of constant $l\left(174: 0\right.$ at right, $175^{\circ}: 0$ at left). 

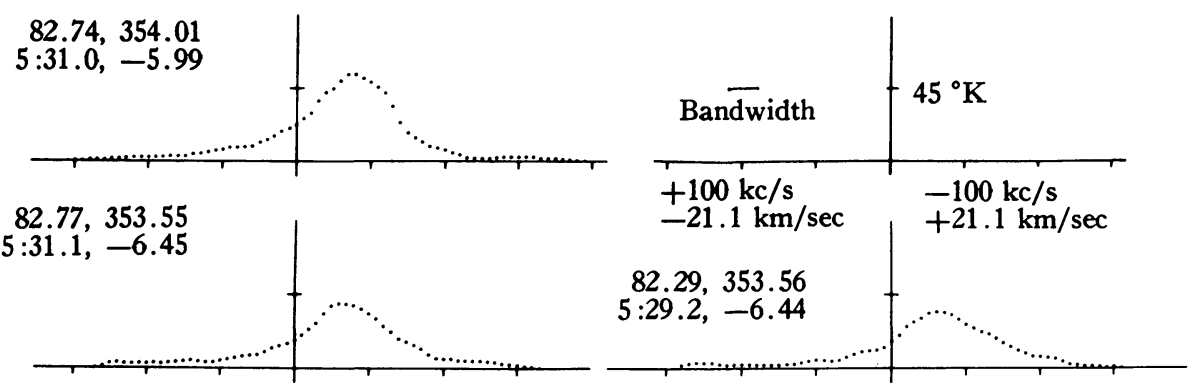

$82.77,353.55$

$5: 31.1,-6.45$

Fig. 3. Profiles obtained in the vicinity of the Orion nebula (right ascension $=83^{\circ}: 32$, declination $=354: 59$ ). At the positions corresponding to the two lower profiles, optical absorption is much less than at the position of the upper left profile (see Fig. 4).

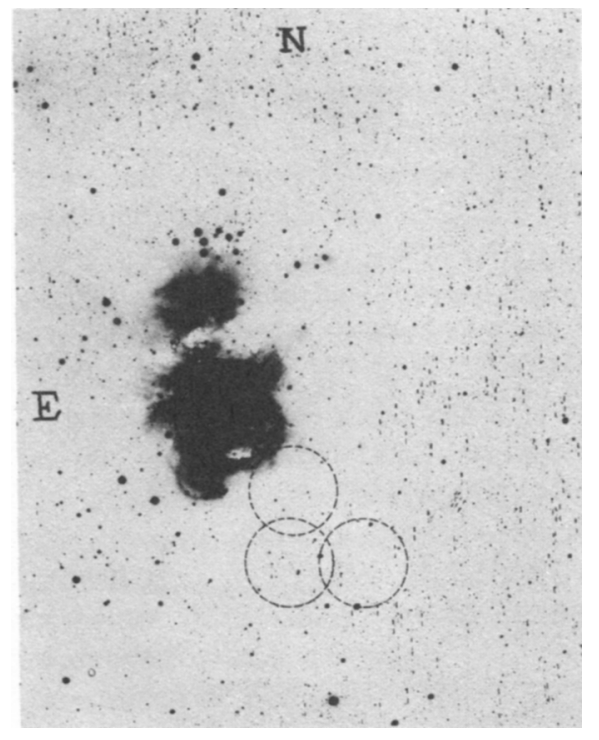

FIG. 4. Lick Observatory photograph of the surroundings of the Orion nebula. The positions of the profiles shown in Fig. 3 are indicated by dashed circles with diameters equal to the telescope beamwidth. Extragalactic systems are marked by small dashes.

Such a correlation between hydrogen intensity and optical absorption is found at many places in Orion, e.g., also in the region of the Horsehead nebula. It is finer and on a smaller scale than the coarse, large-scale correlation found by Lilley [3] in the Taurus region. It stands in marked contrast to the absence of correlation found by Bok, Lawrence, and Menon [4] for dense dark clouds in Ophiuchus and Taurus, and by van de Hulst, Muller, and Oort [5] for dark clouds in Taurus.

The observations were made with financial support from the Netherlands Organization for Pure Research (Z.W.O.) through the Netherlands Foundation for Radio Astronomy. A complete discussion will be published later in B.A.N. 


\section{REFERENCES}

[1] Menon, T. K. Radio Astronomy (I.A.U. Symposium No. 4, 1955). Cambridge, England, 1957, p. 56.

[2] Menon, T. K. Ap. J. 127, 28, 1958.

[3] Lilley, A. E. Ap. J. 121, 559, 1955.

[4] Bok, B. J., Lawrence, R. S., and Menon, T. K. P.A.S.P. 67, 108, 1955.

[5] van de Hulst, H. C., Muller, C. A., and Oort, J. H. B.A.N. 12, 117, 1954 (see page 130).

\section{Discussion}

Graham Smith: I would like to draw attention to recent work by Rishbeth (submitted to M.N.R.A.S.), who used observations from the Mills cross at $85 \mathrm{Mc} / \mathrm{s}$ and other surveys. He presents the available information on the continuum radiation from the Orion region, and estimates the amount of ionized hydrogen in various parts of the region.

van Woerden: I wish to remark that at a later stage of this work a comparison with optical and other radio data will be made. 